See discussions, stats, and author profiles for this publication at: https://www.researchgate.net/publication/323656914

\title{
Uplink Performance Optimization of Ultra Dense Wi-Fi Networks using AP- managed TPC
}

Conference Paper · April 2018

DOI: $10.1109 /$ WD.2018.8361703

CITATION

1

3 authors, including:

Shahwaiz Afaqui

Universitat Oberta de Catalunya

22 PUBLICATIONS 209 CITATIONS

SEE PROFILE

Some of the authors of this publication are also working on these related projects:

Project Digital Predistortion View project

Project Crosstalk Cancelation via Digital Pre-Distortion (DPD) View project
READS

75

Ronan Farrell

National University of Ireland, Maynooth

188 PUBLICATIONS 603 CITATIONS

SEE PROFILE 


\section{Uplink Performance Optimization of Ultra Dense Wi-Fi Networks using AP-managed TPC}

\author{
M. Shahwaiz Afaqui \\ Department of Electronic Engineering \\ Maynooth University \\ Email: Shahwaiz.Afaqui@nuim.ie
}

\author{
Stephen Brown \\ Department of Computer Science \\ Maynooth University \\ Email: Stephen.Brown@nuim.ie
}

\author{
Ronan Farrell \\ Department of Electronic Engineering \\ Maynooth University \\ Email: Ronan.Farrell@nuim.ie
}

\begin{abstract}
In this paper, we study the uplink transmission power control problem in ultra dense Wi-Fi networks and propose two novel access point-controlled frameworks, which determines optimum transmit power settings with the intention of maximizing an objective function. NS-3 simulation results show that the proposed centralized approaches reduce starvation among stations and significantly improves the objective function, resulting in improved performance.
\end{abstract}

\section{INTRODUCTION}

Transmit Power Control (TPC) algorithms can decrease energy consumption and increase performance by reducing MAC-level interference. IEEE 802.11ax provides dynamic adaptation of transmit power for stations that tune their carrier sensing. The new High Efficiency (HE) trigger frames defined by IEEE 802.11ax amendment [1] can allow an AP to control the transmission power of stations.

\section{A. Related work}

The benefits of power control in reducing Co-Channel Interference (CCI) levels is well explored in literature [2], [3]. However, the usage of variable transmit powers for each station in dense networks can result in asymmetric links, and can potentially lead to throughput starvations [4]. Different works have been proposed in literature that utilize power control mechanisms to improve network throughput [5], [6]. In [7], the authors proposed a power control technique which works with an inter-cell coordination to mitigate the interference.

Our work differs by instead of only considering TPC to reduce interference, we propose the use of coordinated adaptation of transmit power (on a per cell basis) that yields increased area throughput and fairness in ultra dense Wi-Fi networks.

\section{B. Contributions}

Through this work, we expose the potential of centralized transmit power control mechanism in improving spatial reuse within high density scenarios. From a detailed simulation study, it is observed that in order to make better decisions on transmit power for a non-AP station, an AP needs to actively monitor and manage the associated stations.

\section{SYSTEM MODEL AND ASSUMPTIONS}

We define ultra dense networks as those in which cells overlap (every AP hears other beacon frames).

\section{A. Network setting}

In our analysis, we consider the scenario defined by the Task Group 802.11ax (TGax) in [8], which consists of a multi-floor residential building (see Figure 1). It included 100 apartments and had the following specifications:

$$
\begin{aligned}
& -5 \text { floors } \\
& -2 \times 10 \text { apartments in each floor } \\
& - \text { Apartment size: } 10 \mathrm{~m} \times 10 \mathrm{~m} \\
& \times 3 \mathrm{~m} \\
& - \text { Residential Building } \\
& \text { - Concrete External wall with } \\
& \text { windows }
\end{aligned}
$$

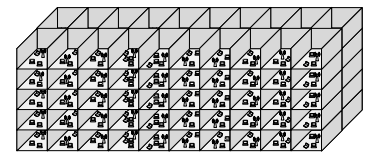

Fig. 1: Ultra dense Wi-Fi deployment in residential building.
A single AP was randomly placed within each apartment. $M$ non-AP stations were randomly placed around each AP. APs select channel 1, 6, and 11 randomly. We study the $2.4 \mathrm{GHz}$ band as it is more restricted in dense environments (only 3 non-overlapping channels).

\section{B. IEEE 802.11 Transmit Power Control}

In our work, we leverage the TPC Request, TPC Report and Power Constraint Information Elements (IE) defined by IEEE $802.11 \mathrm{~h}$ as action frames to exchange link quality information (Received Signal Strength Indication (RSSI) etc.).

\section{Evaluation metrics}

Our schemes use the following metrics:

1) Aggregate Throughput: Good data frame count.

2) Fairness: Jain's fairness index [9] is used.

3) Frame Error Rate (FER): Frame success ratio is used in calculating FER, by counting acknowledgments.

4) End-to-end-delay: The mean delay includes the transmission, queuing and contention delays.

5) Hidden, contending and exposed stations: $P_{X Y}$ is the power of X's transmissions at Y and $S r$ is the sensitivity,

\footnotetext{
Nodes $X, Y$ are hidden if they are not within each other's carrier sensing range $\left(P_{X Y}<C S T_{Y}\right.$ and $\left.P_{Y X}<C S T_{X}\right)$ and a station $\mathrm{Z}$ (intended receiver of either $\mathrm{X}$ or $\mathrm{Y}$ ) is placed within both $X$ 's and $Y$ 's transmission range $\left(P_{X Z}>S r_{Z}\right.$ and $\left.P_{Y Z}>S r_{Z}\right)$.

Nodes $X$ and $Y$ are exposed if they are able to defer each other's transmissions $\left(P_{X Y}>C S T_{Y}\right.$ and $\left.P_{Y X}>C S T_{X}\right)$ but are unable to reach each other's intended receivers $\mathrm{Z} 1$ and $\mathrm{Z} 2\left(P_{X Z 2}<S r_{Z 2}\right.$ and $\left.P_{Y Z 1}<S r_{Z 1}\right)$ respectively.

Nodes $X$ and $Y$ are contending when they are able to defer each other's transmission $\left(P_{X Y}>C S T_{Y}\right.$ and $\left.P_{Y X}>C S T_{X}\right)$.
} 


\section{Objective function}

The goal of transmit power selection for each non-AP station in the uplink transmission is to jointly maximize the achievable throughput with the constraint of airtime fairness, while containing/maintaining the FER and the end-to-end delay for all the cells. Formally, this can be represented by a single objective function:

$$
f=\max \left[\frac{\text { Throughput }_{A g g} \times F_{J}}{\text { Delay }_{A v g} \times F E R_{A v g}}\right]
$$

where Throughput $_{A g g}$ is the aggregated network wide throughput $^{1}, F_{J}$ is the fairness in the network, Delay ${ }_{A v q}$ is the average end-to-end delay and $F E R_{A v g}$ is the average FER of all the links. All APs use this function to find the optimal settings.

\section{ADAPTIVE AP-MANAGED TPC}

\section{A. Fixed percentage based transmit power control}

In this closed-loop method, AP selects a fixed percentage (i.e. $\eta$ ) of stations to enable a decrease in transmit power. Initially, all stations are allowed to transmit data frames at the maximum power level of $16 \mathrm{dBm}$. After a non-AP station receives a TPC Request, it calculates the average RSSI of received beacon frames before the Updateperiod (which is a multiple of beacon interval time) and reports it to the associated AP. Based on the RSSI or link margin, the AP ranks and selects the percentage of stations that have the highest RSSI. After each Updateperiod, $\eta$ percentage of stations with best AP-to-station link quality are allowed to reduce their transmit power by step size $\Delta$ up till the $P_{\min }$ value is attained. No station is allowed to reduce power below $P_{\min }$.

For the selected stations to guarantee the target data rate, the values of $P_{\min }$ is set based on:

$$
P_{\text {min }}>D_{k}+S_{k}
$$

where $D_{k}$ is the path loss for the uplink (calculated using Equation 4) and $S_{k}$ is the AP receiving sensitivity for the target data rate In our evaluation, the AP varies the $P_{\min }$ to find the optimal value that results in the maximization of $f$.

\section{B. Margin based transmit power control}

In this closed loop method, each station independently calculates the transmit power based on the RSSI of beacons of the associated AP. Stations placed nearer to the AP reduce power which reduces exposed nodes and stations placed at furthest distance use maximum power which decreases the hidden nodes. The baseline mechanism to set transmit power for a non-AP station $k$ is calculated by:

$$
P_{T X k}=\text { Margin }-D_{K}-S_{k}
$$

Assuming symmetric uplink and downlink measurements, nonAP stations estimates $D_{k}$ based on the actual transmitted power and the received beacon power based on:

$$
D_{k}=P_{R X k_{t-1}}-P_{T X k_{t-1}}
$$

where $P_{T X k_{t-1}}$ and $P_{R X k_{t-1}}$ are the transmission power level and the received power of a beacon frame before the Updateperiod at station $k$. Using equations 3 and 4, a station

\footnotetext{
${ }^{1}$ Aggregate throughput only does not account for how resources are shared among different clients
}

TABLE I: Physical and MAC layer parameters for simulation.

\begin{tabular}{|c|c||c|c|}
\hline Parameter & Values & Parameter & Values \\
\hline Wireless Standard & IEEE802.11n & Packet size & 1000 bytes \\
\hline No. of BSS & 32 & No. of client per AP & 16 \\
\hline Frequency band & $2.4 \mathrm{GHz}$ & $\begin{array}{c}\text { Transmission power of } \\
\text { STA and AP }\end{array}$ & $16 \mathrm{dBm}$ \\
\hline $\begin{array}{c}\text { Physical transmission } \\
\text { rate }\end{array}$ & $\begin{array}{c}\text { MCS 7 for data, MCS 0 for } \\
\text { Control/management }\end{array}$ & Antenna gain & $1 \mathrm{~dB}$ \\
\hline Propagation loss model & Hybrid buildings & Noise figure & $7 \mathrm{~dB}$ \\
\hline Wall penetration loss & $12 \mathrm{~dB}$ & Fading model & not used \\
\hline Floor penetration loss & $17 \mathrm{~dB}$ & AP receiving sensitivity & $65 \mathrm{dBm}(\mathrm{MCS} 7)$ \\
\hline Guard interval & Short & Data preamble & Short \\
\hline Channel width & $20 \mathrm{MHz}$ & Beacon Interval & $100 \mathrm{~ms}$ \\
\hline Aggregation & not used & RTS/CTS & disabled \\
\hline
\end{tabular}

can calculate its transmit power. where $S_{k}$ is the AP receiving sensitivity for the target data rate for station $k$. The value of Margin is pre-defined by the AP and is selected based on active learning. The selected power is confined between the minimum $\left(P_{\min }\right)$ and maximum $\left(P_{\max }\right)$ supported power levels:

$$
P_{T X k}=\min \left(\max \left(P_{T X k}, P_{\min }\right), P_{\max }\right)
$$

\section{Simulation Setup}

The simulations were carried out using the NS-3 network simulator in which the Hybrid Building propagation loss model was used [10]. For the final calculated results, a large enough number of simulations were run in order to achieve $95 \%$ confidence intervals (a minimum of 12 runs for each case and the simulation time was 45 seconds). We considered uplink transmission $^{2}$, where each station was in saturation condition ${ }^{3}$ The data rate used for each non-AP station is 3 Mbps. The Physical and MAC layer parameters are shown in Table I.

\section{Simulation Results AND DisCUSSION}

In the following results, we compare the proposed algorithms with a legacy IEEE 802.11 network, where all nonAP stations utilize fixed transmit power (i.e. $16 \mathrm{dBm}$ ). Apart from Section V-C, all the stations employ a fixed MCS (as highlighted in Table I

\section{A. Evaluating fixed percentage based TPC}

The objective function value for simulations using constant transmit power is 0.386 . An AP gradually increases the number of stations (i.e. $0.125,0.25, \ldots, 0.875 \%$ ) to reduce the transmit power by a fixed step size of $\Delta$ of $2 \mathrm{~dB}$ up till $P_{\min }$ values (i.e. 2, 6, 12 and $14 \mathrm{dBm}$ ). During simulations, after each Updateperiod (i.e. 2 seconds ${ }^{4}$ ), the AP selects $\eta$ percentage of stations to reduce the transmit power. Figure 2 gives substance to the idea of intelligently selecting the best combination. In our simulations, the optimal value of the objective function (i.e. 0.48389$)$ is achieved when 12 stations $(0.75 \%)$ in each cell reduce their transmit power to $10 \mathrm{dBm}$. Figure $2 \mathrm{~b}$ indicates fair throughput improvements of near $11 \%$ and Figure 4a shows that this technique results in fewer contending stations.

\section{B. Evaluating margin based transmit power control}

After the completion of Updateperiod of 2 seconds, each station calculates the target transmit power based on Equation 3. The selected value is confined between a $P_{\min }$ value of 1 and $P_{\max }$ value of $16 \mathrm{dBm}$. The objective function value

\footnotetext{
${ }^{2}$ We evaluate the performance over uplink transmissions because it is the worst case in terms of contention.

${ }^{3}$ Saturation is used to explore maximum capacity.

${ }^{4}$ The convergence time can be lowered to accommodate few beacon intervals for best performing links
} 

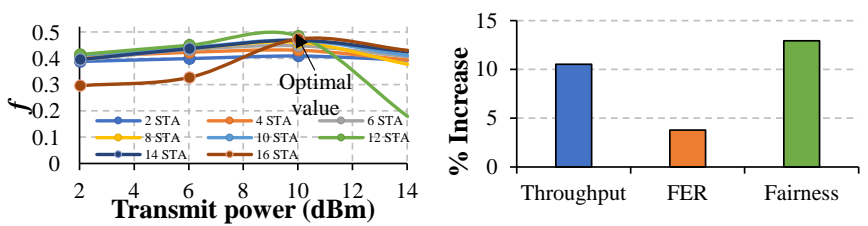

(a) Objective function versus (b) Performance at the optimal transmit power. value.

Fig. 2: Performance improvements of AP-managed fixed percentage TPC
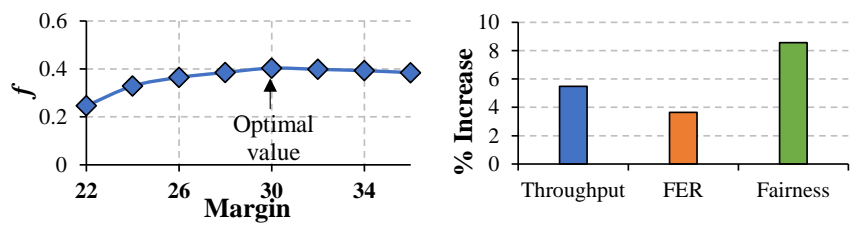

(a) Objective function versus (b) Performance at the optimal transmit power. value.

Fig. 3: Performance improvements of margin based TPC achieved for simulations using constant transmit power is 0.3703 . Figure $3 \mathrm{a}$ shows the optimal value (i.e. 0.4033 ) at a Margin of 30, where more than 5\% throughput and near $11 \%$ increase in fairness is achieved. According to Figure $4 b$, an important outcome of this technique is a considerable decrease in hidden, exposed and contending stations.

\section{Impact of fixed percentage based transmit power control on a network employing rate adaptation}

With the help of Figure 5, we illustrate the impact of fixed percentage based transmit power control on an IEEE 802.11n networks with Minstrel $^{5}$ [11] rate adaption algorithm. As expected, AP managed TPC improves the performance of the network also in the presence of rate adaptation $(15 \%$ increase in throughput and 35\% increase in fairness). The same improvements are also expected for the proposed margin based transmit power control algorithm.

\section{Discussion}

Results indicate that an increase in transmission opportunity (with a decrease in contending stations) results in a slight increase in network wide FER. However, both the techniques resulted in considerable fairness improvements. Moreover, results indicate that instead of hidden or exposed station, any TPC technique designed with the aim of reducing the contending stations will result in network wide improvements.

Results for both the schemes indicate the stability of the system, where absolute limit retains the power levels for each station. While margin based scheme is trivial to implement, percentage based scheme results in greater improvements in the objective function. However, the short coming of percentage based scheme is the optimization time required to select the number of stations and their respective transmit powers.

\section{CONCLUSION}

In this paper, we propose a novel AP-managed adaptive per-link transmit power control approach. Two mechanisms are evaluated in ultra dense IEEE 802.11 environments by

\footnotetext{
${ }^{5}$ Minstrel is the default rate control in Linux (for NICs supporting soft-MAC through mac80211 kernel module).
}

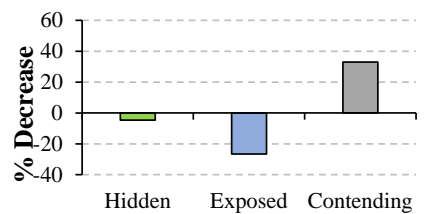

(a) Fixed percentage based TPC

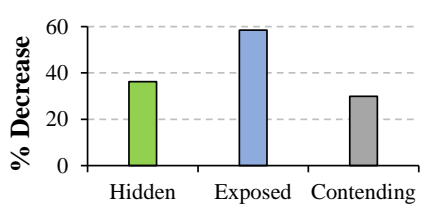

(b) Margin based TPC
Fig. 4: Impact on channel conditions

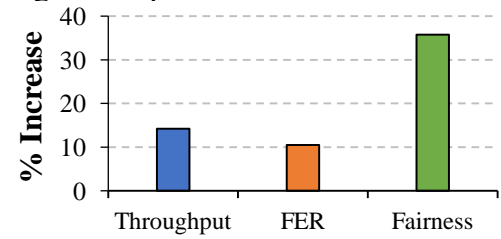

Fig. 5: Improvements provided by fixed percentage based transmit power control with rate adaptation.

using an objective function. Results reveal that the proposed closed-loop mechanisms provide considerable improvements (more than $10 \%$ in throughput and fairness) by systematically reducing the number of contending station. In addition, the proposed scheme was also observed to improve substantially the throughput of the systems that implement adaptive rate control. The significance of this work is to identify AP controlled TPC methods that can improve spatial reuse and fairness in extremely dense networks.

\section{ACKNOWLEDGEMENTS}

This publication has emanated from research conducted with the financial support of Science Foundation Ireland (SFI) and is co-funded under the European Regional Development Fund under Grant Number 13/RC/2077.

\section{REFERENCES}

[1] M. S. Afaqui, E. Garcia-Villegas, and E. Lopez-Aguilera, "IEEE 802.11ax: Challenges and requirements for future high efficiency WiFi," IEEE Wireless Communications, no. 99, pp. 2-9, 2017.

[2] M. Krunz, A. Muqattash, and S.-J. Lee, "Transmission power control in wireless ad hoc networks: challenges, solutions and open issues," IEEE Network, vol. 18, no. 5, pp. 8-14, Sept 2004.

[3] D. Qiao, S. Choi, A. Jain, and K. G. Shin, "Adaptive transmit power control in IEEE 802.11a wireless LANs," in VTC Spring., vol. 1, April 2003, pp. 433-437 vol.1.

[4] V. P. Mhatre, K. Papagiannaki, and F. Baccelli, "Interference mitigation through power control in high density 802.11 wlans," in IEEE INFOCOM, May 2007, pp. 535-543.

[5] K. P. Shih, Y. D. Chen, and C. C. Chang, "A physical/virtual carriersense-based power control mac protocol for collision avoidance in wireless ad hoc networks," IEEE Transactions on Parallel and Distributed Systems, vol. 22, no. 2, pp. 193-207, Feb 2011.

[6] D. N. M. Dang, C. S. Hong, S. Lee, and J. Lee, "A sinr-based mac protocol for wireless ad hoc networks," IEEE Communications Letters, vol. 16, no. 12, pp. 2016-2019, December 2012.

[7] O. Oteri, P. Xia, F. LaSita, and R. Olesen, "Advanced power control techniques for interference mitigation in dense 802.11 networks," in WPMC, June 2013, pp. 1-7.

[8] S. Merlin, "IEEE 802.11 TGax simulation scenarios," IEEE 802.11ax, IEEE 802.11-14/0621r3.

[9] R. Jain, "Fairness: How to measure quantitatively?" ATM Forum/940881, Sept. 1994.

[10] Hybrid buildings propagation loss model: ns3-design document. [Online]. Available: http://www.nsnam.org/docs/models/html/buildingsdesign.html

[11] D. Xia, J. Hart, and Q. Fu, "On the performance of rate control algorithm minstrel," in IEEE PIMRC, Sept 2012. 